\title{
Erratum to: Examining the Role of Social Feedbacks and Misperception in a Model of Fish-Borne Pollution Illness
}

\author{
Michael Yodzis
}

\section{Erratum to:}

(C) Springer International Publishing Switzerland 2016

J. Bélair et al. (eds.), Mathematical and Computational Approaches in Advancing Modern Science and Engineering, DOI 10.1007/978-3-319-30379-6_32

\section{Erratum DOI 10.1007/978-3-319-30379-6_71}

The authorship of the present article has been revised so that Michael Yodzis is now listed as the only author, to make clear that Dr. Chris T. Bauch and Dr. Madhur Anand were not contributors to this publication. The present contribution is an extended and revised version of the publication 'Coupling fishery dynamics, human health and social learning in a model of fish-borne pollution exposure' by M. Yodzis, C.T. Bauch and M. Anand, Sustainability Science 11, pp. 179-192 (2016).

The results are extended to include a more complete explanation of the model's dependence on its initial conditions, and the sensitivity analysis is enlarged to study the influence of the ecological parameters on the model's qualitative outcomes. Any mistakes or omissions are the sole responsibility of Michael Yodzis.

The updated online version of the original chapter can be found at DOI 10.1007/978-3-319-30379-6_32 